\section{Addressing a patient-controlled approach for genomic data sharing}

To the Editor: We appreciate the recent statement in Genetics in Medicine "Laboratory and Clinical Genomic Data Sharing Is Crucial to Improving Genetic Health Care: A Position Statement of the American College of Medical Genetics and Genomics". ${ }^{1}$ We support the position of ACMG and strongly agree that sharing of genomic information is becoming increasingly important for the care of individual patients and understanding of disease pathways. In response to this statement, we want to elaborate on a specific mechanism for which data sharing can be considered: a patient-controlled approach. In addition, we want to discuss several challenges that must be addressed to achieve patient sharing of genomic data.

The patient-controlled approach has shown its promise although more research and discussion is needed. A recent study, and several others, revealed that research participants strongly support obtaining their genetic test results, expressing a desire of ownership of their genetic information, mostly for ease in sharing with health providers or family members, as well as a strong belief in patient empowerment. ${ }^{2}$ Currently, there are several initiatives (e.g., GenomeConnect, My Research Legacy by the American Heart Association, and numerous National Institutes of Health registries) that invite patients to share biomedical and genetic data for research and health purposes. Additionally, the National Institutes of Health and Office of the National Coordinator for Health Information Technology recently launched Sync 4 Science, a pilot program that aims to give patients an easy way to share their health data with researchers in support of the goal of the All of $U s^{S M}$ Research Program. Consistent with the US Department of Health and Human Services' vision in health information technology, there is an increased emphasis on empowering patients to control and share their healthcare data with providers and/or researchers.

There are several reasons that patients may wish to access and share their genetic information, many of which were defined recently in a personal communication when the American Civil Liberties Union filed a complaint pursuant to the Health Insurance Portability and Accountability Act with the US Department of Health and Human Services. Specifically, four patients were denied their requests for all of their genetic sequencing information from a well-known supplier of genetic testing. Rehm, in her letter of support, defends the right of patients to access their genomic data for several incentives, one of which is that patients may wish to advance research via data sharing of their own. ${ }^{3}$ This
American Civil Liberties Union complaint highlights the current lack of guidelines and standards regarding patients' access and rights to their own genetic data and also highlights the desire of individuals to understand their healthcare data and become more active partners in their health.

Genetic information about an individual inherently poses risk about privacy and confidentiality. For example, the potential predictive power of certain genetic variants in a person poses a confidentiality risk from unwanted use or disclosure of health status. To prevent discrimination on the basis of this type of genetic information, US Congress passed the Genetic Information Nondiscrimination Act of 2008. Additional efforts, as we advocate here, towards development of a patient-controlled approach for genomic data sharing would enable individuals to set control over their own sharing, privacy, and consent preferences. If patients could control data sharing, many of the ethical and regulatory issues concerning sharing of genomic data could be directly addressed or alleviated.

Regardless of patient- or institute-controlled approaches, the sheer volume and complexity of genomic data can also be a barrier to sharing. The majority of Clinical Laboratory Improvement Amendments-certified laboratories (e.g., Baylor Miraca Genetics Laboratories) providing clinical genome sequencing services and even companies that offer direct-toconsumer genetic testing (e.g., 23andMe and Genos) do provide raw sequence data upon request in the form of .fastq, .bam, and/or .vcf files via either secure online transfer or portable hard drives. However, many labs restrict raw data requests to only the ordering clinician and not patients, making it nearly impossible for a patient to access and share their own genetic data either with other healthcare providers at unaffiliated institutions or into public databases. Besides, transfer of large files online may be technologically challenging for some patients, while shipping of hard drives can be burdensome and costly (approximately 150-200 USD) to a patient. It is imperative that we change the system to technologically accommodate efficient and secure storage and transport of genomic data. We support the Office of the National Coordinator roadmap that encourages those involved in health information technology to contribute to development of a defined, shared roadmap leveraging health information technology interoperability to ultimately protect and advance healthcare for all.

There is a massive potential in research and clinical settings to leverage genomic data to advance human health overall. This potential, however, comes with many challenges. Efforts towards developing a patient-controlled approach for sharing of personal genome data will undoubtedly contribute to research and clinical initiatives. Ultimately, this type of approach will benefit the sake of patient engagement and will promote the following: (i) data sharing across health organizations for clinical care purposes and/or (ii) contribution of data to public databases for research purposes. 


\section{DISCLOSURE}

The authors declare no conflict of interest.

\section{Katherine E. Miller, $P h D$ and Simon M. Lin, MD, MBA}

Research Information Solutions and Innovation, The Research Institute at Nationwide Children's Hospital, Columbus, Ohio, USA. Correspondence: Simon M. Lin (Simon.Lin@nationwidechildrens.org)

\section{REFERENCES}

1. ACMG Board of Directors. Laboratory and clinical genomic data sharing is crucial to improving genetic health care: a position statement of the American College of Medical Genetics and Genomics. Genet Med 2017. e-pub ahead of print 5 January 2017.

2. Wright MF, Lewis KL, Fisher TC, et al. Preferences for results delivery from exome sequencing/genome sequencing. Genet Med 2014;16:442-447.

3. Rehm HL. Letter of support-Our genes, our data: patients' right to access their own genetic information. US Department of Health and Human Services, 2016

Advance online publication 20 April 2017. doi:10.1038/gim.2017.36

\section{Response to de Vries et al.}

To the Editor: In their paper "Pompe disease in adulthood: effects of antibody formation on enzyme replacement therapy", ${ }^{1}$ de Vries et al. describe their study of a cohort of 73 adult Pompe disease patients who received enzyme replacement therapy (ERT) with alglucosidase alfa for a median duration of 35 months. Patients were classified into high, intermediate and none/low titer groups based on their highest antibody titer. Using muscle strength, pulmonary function and in vitro neutralization assays, the authors concluded that antibody titers had limited interference with ERT efficacy. It was proposed that patients with the IVS1/delex18 GAA genotype may have an attenuated antibody response. We commend the authors' efforts as this study adds to the existing knowledge about clinical course and outcomes in late-onset Pompe disease (LOPD). However, we feel that some conclusions need to be drawn with caution as there are limitations of this study, namely: (i) the classification of patients based on a single peak antibody titer; (ii) the clinical endpoints used for outcome assessment; (iii) the role of neutralizing versus non-neutralizing antibodies in predicting clinical outcome; and (iv) genotype correlation with immune response.

The approval of alglucosidase alfa in 2006 was a breakthrough in the treatment of Pompe disease. However, this therapeutic protein has faced challenges of immunogenicity, with the development of high-sustained antibody titers at one extreme and no/low titers at the other. Recently, there has been an increase in the understanding of the impact of sustained intermediate titers on ERT efficacy. The Myozyme package insert documents that patients with antibody titers greater than or equal to 12,800 had a $50 \%$ increase in enzyme clearance from week 1 to week 12 of treatment. The negative impact of high-sustained antibody titers and sustained intermediate titers is well established in patients with infantile Pompe disease (IPD), who have lower ventilator-free and overall survival and show deterioration in other measures, such as left ventricular mass index, gross motor development and urinary glucose tetrasaccharide when compared to IPD patients with low antibody titers. ${ }^{1}$ We have seen that some of our IPD patients with overall low antibody titers may have a single high titer value, but eventually have good clinical outcomes compared with those who have high-sustained antibody titers or sustained intermediate titers. Our experience shows that it is the persistence and trend of the immune response over time that is closely related to treatment outcome, rather than a single peak antibody titer. Therefore, it is important to reclassify patients in this published study as "high sustained" instead of "high" titer, and, "sustained intermediate" instead of "intermediate" titer, as the current classification based on a single, maximal value may lead to lack of clarity of the role of antibody titers.

Neutralizing antibodies inhibit enzyme activity by at least two mechanisms: inhibiting enzyme uptake by cells and/or inhibiting catalytic activity. It is noteworthy that even in cross-reactive immunologic material (CRIM)-negative IPD patients with high-sustained antibody titers, only a subset demonstrated neutralizing antibodies in in-vitro assays.,3 Even among those who had neutralizing antibodies, some demonstrated antibodies only to the catalytic domain and not to the uptake domain. However, irrespective of the presence/ type of neutralizing activity, they all experienced poor clinical outcomes. The role of non-neutralizing antibodies should not be overlooked because, when present in high titers, they can reduce the efficacy of ERT by altering its biodistribution; for instance, into Fc receptor-expressing cells and hepatic uptake of complement-bound soluble immune complexes. Consequently, the inability to detect neutralizing activity by in vitro assays should not lead to conclusions about availability of enzyme activity in-vivo since in-vitro measurement does not always reflect the true in-vivo situation.

Another factor contributing to patient classification is the performance of the assays, since assay variability may affect how patients were grouped. The reported percentage coefficient of variation appears to be associated with control reagents with no specific information on the reproducibility of titering patient samples. Neutralizing antibody was detected by measuring enzyme activity both in the medium directly after enzyme addition and before cell harvest, while activity incorporated was measured in cell homogenates. Standardization of this type of measure and the variability that could be encountered by a cellular matrix is not addressed. Overall, repeat measures over time, such as looking at the persistence of antibody titers and including this temporal aspect, could better align the way patients are categorized based on antibody responses.

The study detected a very limited association between antibody titers and clinical outcomes. In IPD, with its dramatic presentation, clinical endpoints (such as left 
ventricular mass/left ventricular mass index, ventilator-free survival or overall survival) are easy to measure and observe. Clinical outcome measures used to assess ERT response were the Medical Research Council scale and forced vital capacity. In a slowly progressive disease such as LOPD, such distinct end points take years to be evident and these measures may not be sufficiently sensitive to capture the gradual change. Moreover, these metrics may not be able to differentiate between the impact of natural disease progression versus the age-related decline in function. The Medical Research Council scale has long been criticized for its limitations, and there have been numerous attempts to improve its accuracy.

The authors speculate that the IVS1/delex18 genotype may protect against developing high antibody titers. It is known that genotype alone does not predict ERT response in Pompe disease. A number of factors, such as major histocompatibility complex class II polymorphisms, human leukocyte antigen haplotypes, the extent of non-endogenous epitopes relative to ERT, and epitope spreading (which may lead to high titers), may play a role in the treatment response. In our experience, a small fraction of CRIM-negative IPD patients do not develop high antibody titers and respond favorably to ERT, which suggests that genotype alone is not responsible for the immune response. ${ }^{4}$ Thus, the observation of genotype association in LOPD should be stated with caution.

In summary, we believe that the persistence of elevated titers over time, rather than the absolute values at a single time point, is a key predictor of clinical outcomes. It remains to be examined whether a complete elimination of antibody formation from the time of ERT initiation would change the outcome. Outcome measures that have the ability to capture small changes in LOPD need to be developed.

\section{DISCLOSURE}

P.S.K. has received research/grant support from Sanofi Genzyme, Valerion Therapeutics, Shire Pharmaceuticals, Alexion, the National Institutes of Health, the Food and Drug Administration, and the Patient-Centered Outcomes Research Institute. She also receives consulting fees and honoraria from the Sanofi Genzyme, Shire Pharmaceuticals, Alexion Pharmaceuticals, Amicus Therapeutics and Roche. She serves as a member of the Pompe and Gaucher Disease Registry Advisory Board for Sanofi Genzyme, and is a Registry Board Member for Alexion Pharmaceuticals. She also serves on the Data Safety Monitoring Board for PTC Therapeutics. S.R. is an employee of Sanofi and owns stock in Sanofi. The other authors declare no conflict of interest.

\section{Mrudu Herbert, $M D^{1}$, Zoheb B. Kazi, $M B B S^{1}$, Susan Richards, $P h D^{2}$, Amy S. Rosenberg, $M D^{3}$ and Priya S. Kishnani, $M D^{1}$}

${ }^{1}$ Division of Medical Genetics, Department of Pediatrics, Duke University Medical Center, Durham, North Carolina, USA; ${ }^{2}$ Translational Medicine and Early Development, Sanofi, Framingham, Massachusetts, USA; ${ }^{3}$ Division of Therapeutic Proteins, Office of Biotechnology Products, Center for Drug Evaluation and Research, US Food and Drug Administration, Bethesda, Maryland, USA. Correspondence: Priya S. Kishnani (Priya.kishnani@duke.edu)

\section{REFERENCES}

1. de Vries JM, Kuperus $E$, Hoogeveen-Westerveld $M$, et al. Pompe disease in adulthood: effects of antibody formation on enzyme replacement therapy. Genet Med 2017;19:90-97.

2. Banugaria SG, Prater SN, Ng YK, et al. The impact of antibodies on clinical outcomes in diseases treated with therapeutic protein: lessons learned from infantile Pompe disease. Genet Med 2011;13:729-736.

3. Kishnani PS, Corzo D, Nicolino M, et al. Recombinant human acid [alpha]glucosidase: major clinical benefits in infantile-onset Pompe disease. Neurology 2007;68:99-109.

4. Berrier $\mathrm{KL}$, Kazi ZB, Prater $\mathrm{SN}$, et al. CRIM-negative infantile Pompe disease: characterization of immune responses in patients treated with ERT monotherapy. Genet Med 2015;17:912-918.

Advance online publication 11 May 2017. doi:10.1038/gim.2017.48

\section{Response to Herbert et al.}

To the Editor: We thank Herbert et al. ${ }^{1}$ for their interest in our work. ${ }^{2}$ Their laboratory has shown to be instrumental in studying the effects of enzyme replacement therapy (ERT) in infants with Pompe disease. However, there are some misunderstandings about our study on adult Pompe patients and antibody formation. Below we explain these in detail.

Herbert et al. ${ }^{1}$ suggest that our patients be reclassified based on sustained titers rather than peak titers, because "the current classification based on a single, maximal value may lead to a lack of clarity on the role of antibody titers". We agree that the duration of high neutralizing antibody titers is important to consider. This is why we measured titers at multiple time points over a period of 3 years (Figures 1 and 3 , and Supplementary Figure $1 \mathrm{~A}-\mathrm{C}$ in de Vries et al. ${ }^{2}$ ). We observed two trends: (i) a decline of peak titers over this period at a group level and (ii) relatively few patients with high $(\geq 31,250)$ peak antibody titers (16 of $73 ; 22 \%)$. Nine $(12 \%)$ of these had high sustained antibody titers. Eight patients $(11 \%)$ had very high $(\geq 156,250)$ peak titers, and these classified for all but one patient as sustained high. This shows that no matter how the groups are generated, in all of these cases group sizes are very small. The statistical power to analyze potential effects on clinical outcome is limited. Therefore, we have also analyzed the eight patients with a very high peak titer and seven patients with a high sustained titer on an individual basis, and we concluded that antibodies were likely to have interfered with the effect of ERT in only one patient. We previously reported on the counteracting effect of high sustained antibodies in this particular patient. ${ }^{3}$ Herbert and colleagues may have missed the fact that only a few adult patients develop high sustained antibodies, which is in contrast to the situation in classic infantile patients. A recent study by Masat et al. ${ }^{4}$ on behalf of the French Pompe Registry Study Group also concluded that antibodies are not a major concern in adults with Pompe disease. ${ }^{4}$ 
Herbert et al. ${ }^{1}$ note that not all cross-reactive immunologic material-negative infantile Pompe patients developed neutralizing antibodies, while they all experienced poor clinical outcome, and that the role of neutralizing antibodies should not be overlooked. We agree that neutralizing antibodies are not the only explanation for a poor response to ERT. First, ERT does not compensate in all cases for $\alpha$-glucosidase (GAA) deficiency to an activity level above the critical threshold. The reason for this could be that the dosage is too low or the therapy is inefficient due to the formation of antirecombinant human GAA antibodies, which neutralize GAA activity and/or interfere with cellular uptake. Second, Pompe disease may have progressed too far and tissue damage has become beyond repair. Third, as yet unknown modifying factors may enhance or decrease the effect of ERT. Fourth, the lysosomal storage of glycogen in Pompe disease induces secondary cellular responses, such as a block of autophagic flux and mitochondrial dysfunction-processes bound to interfere with ERT. Evidently, antibodies are just one of several factors determining the outcome of ERT. This is also emphasized by the heterogeneous response to ERT in patients with no or low antibody titers in our study.

Herbert and colleagues ${ }^{1}$ suggest that assay variability "appears to be associated with control reagents" rather than titering patient samples. It is unclear to us why the authors conclude this as this is misconstrued from our paper; we did use patient samples over the titer range to determine assay variability.

Herbert et al. ${ }^{1}$ question whether the assay used in our study to measure neutralizing effects has been standardized and whether the cellular matrix could cause variability. The assay has been standardized and the same cellular matrix (fibroblasts from a classic infantile patient without any detectable GAA activity) was used in all experiments. We would like to emphasize that assessment of neutralizing effects is an important aspect to investigate the potential impact of antibodies on ERT, and we wish to promote its assessment as a standard assay whenever high antibody titers are found.

The authors also question the use of our clinical outcome measures as a readout for efficacy. We note that the outcome measures have been internationally recognized in consensus meetings and have been found suitable for the detection of changes in patient performance in response to ERT in multiple clinical studies. We recommend testing for the presence of neutralizing antibodies in the case of infusionassociated reactions and when clinical outcome declines.

Herbert et al. ${ }^{1}$ state that "genotype alone is not responsible for immune response" and that "the observation of genotype association in LOPD should be stated with caution". We regret what appears to be a misunderstanding of our work. We did not state in our article that genotype alone is responsible for the immune response. We did, however, state that our results should be confirmed in a larger patient group.

In summary, we have conducted an in-depth study in which we measured antibody titers and their neutralizing effects at multiple time points over a period of 3 years. This showed that titers declined on a group level, a limited number of patients developed high antibody titers, and a subset of these patients showed high sustained titers, but in only one patient was a clear impact of antibodies on the effect of ERT likely.

\section{DISCLOSURE}

A.T.vdP. has provided consulting services for various industries in the field of Pompe disease under an agreement between these industries and Erasmus MC. The other authors declare no conflict of interest.

Juna M. de Vries, $M D, P h D^{1,2,6}$, Esther Kuperus, $M D^{1,2,6}$, Marianne Hoogeveen-Westerveld, $B S c^{1,3}$,

Marian A. Kroos, BSc ${ }^{1,3}$, Stephan C.A. Wens, $M D^{1,2}$,

Merel Stok, PhD ${ }^{1,3,4}$, Nadine A.M.E. van der Beek, MD, PhD ${ }^{1,2}$, Michelle E. Kruijshaar, $P h D^{1,4}$, Dimitris Rizopoulos, $P h D^{5}$,

Pieter A. van Doorn, $M D, P h D^{1,2}$,

Ans T. van der Ploeg, $M D, P h D^{1,4}$ and

W.W.M. Pim Pijnappel, PhD ${ }^{1,3,4}$

${ }^{1}$ Center for Lysosomal and Metabolic Diseases, Erasmus MC University Medical Center, Rotterdam, The Netherlands; ${ }^{2}$ Department of Neurology, Erasmus MC

University Medical Center, Rotterdam, The Netherlands; ${ }^{3}$ Molecular Stem Cell Biology, Department of Clinical Genetics, Erasmus MC University Medical Center, Rotterdam, The Netherlands; ${ }^{4}$ Department of Pediatrics, Erasmus MC University Medical Center, Rotterdam, The Netherlands; ${ }^{5}$ Department of Biostatistics, Erasmus MC University Medical Center, Rotterdam, The Netherlands. Correspondence: W.W.M. Pim Pijnappel (w.pijnappel@erasmusmc.nl)

${ }^{6}$ The first two authors contributed equally to this work.

\section{REFERENCES}

1. Herbert M, Kazi ZB, Richards S, et al. Response to de Vries et al. Genet Med advance online publication, May 11, 2017; doi:10.1038/ gim.2017.48.

2. de Vries JM, Kuperus E, Hoogeveen-Westerveld M, et al. Pompe disease in adulthood: effect of antibody formation on enzyme replacement therapy. Genet Med 2017;19:90-97.

3. de Vries JM, van der Beek NA, Kroos MA, et al. High antibody titer in an adult with Pompe disease affects treatment with alglucosidase alfa. Mol Genet Metab 2010;101:338-345.

4. Masat E, Laforêt P, De Antonio M, et al. Long-term exposure to Myozyme results in a decrease of anti-drug antibodies in late-onset Pompe disease patients. Sci Rep 2016;6:36182.

\section{Pitfalls of trio-based exome sequencing: imprinted genes and parental mosaicism-MAGEL2 as an example}

To the Editor: Family-based whole-exome sequencing has proven to be an effective diagnostic strategy for the identification of causative variants in individuals with intellectual disability (ID) and congenital malformations 
(CM). De novo mutations play a major role in ID/CM and it is estimated that they are responsible for up to $40 \%$ of cases in non-consanguineous populations. ${ }^{1}$ Most whole-exome sequencing filtering strategies applied in laboratories worldwide are focused on de novo, X-linked and recessive inheritance. However, in their article "Imprinting: The Achilles Heel of Trio-Based Exome Sequencing," Aten et al. ${ }^{2}$ recently highlighted the importance of taking into account mutations in imprinted genes as a cause of ID. They described the difficulties they confronted in the identification of the causative variant in a large family with several affected members. The family was studied in parallel in two independent centers using different diagnostic approaches. A trio-based approach was used for one part of the family and single-exome sequencing for another member; both failed to identify the pathogenic mutation. Only when the analysis of the family pedigree showed that all affected individuals were linked through their fathers was the causative variant, a paternally inherited frameshift mutation in MAGEL2, identified. Truncating mutations in the paternally expressed allele of MAGEL2, located in the imprinted 15q11q13 Prader-Willi syndrome region, are responsible for Schaaf-Yang syndrome. ${ }^{3}$ An additional pitfall when applying the common filtering strategies may be encountered if parental mosaicism is present. Recent unpublished data from the Deciphering Developmental Disorders study estimate that around 2\% of pathogenic de novo mutations in children are mosaic in parental tissues.

We report a seven-year-old boy initially referred to our clinic at 3 years of age for clinical evaluation. He is the second child of a non-consanguineous healthy couple with no family history of note. His elder brother is healthy. Pregnancy was uneventful (normal fetal movements) and delivery was at term by $\mathrm{C}$-section due to breech presentation. The birth weight was $2,830 \mathrm{~g}$ ( 15 th centile). In the neonatal period, hypotonia, poor suck and scarce spontaneous movements were noted. On examination at 3 years of age, the patient showed marked growth delay (height: -4 s.d., weight: -3 s.d., occipitofrontal circumference: -2.5 s.d.). He had dry skin and an abnormal hair growth pattern. Dysmorphic features consisted of dolicocephaly, low-set ears, a broad nasal root, a deep philtrum and widely spaced teeth. He had mild contractures of both knees, tapering digits with camptodactyly of fingers 2 to 5 , and poorly developed palmar creases. He had male genitalia with a hypoplastic penis and scrotum. Bilateral cryptorchidism had been surgically corrected. His psychomotor development was markedly delayed: he was unable to walk independently, speech was almost absent, and he was just able to comprehend simple orders. He had suffered two seizures (normal magnetic resonance imaging and electroencephalogram) and was undergoing gastroenterology and endocrine follow-up due to chronic constipation and recurrent hypoglycemias of unknown origin. No clinical diagnosis could be established. Initial genetic testing included a karyotype, a custom-designed $60 \mathrm{~K}$ oligonucleotide array (KaryoArray v3.0) and the CytoSNP-850 K Beadchip
(Illumina), all with normal results. Subsequent trio exome analysis using a de novo filtering approach revealed an apparently de novo heterozygous frameshift mutation in MAGEL2 (NM_019066.4):c.1996dupC (p.Gln666fs) previously described in other patients with Schaaf-Yang syndrome. Sanger sequencing validation confirmed the variant in the proband and enabled us to identify the same frameshift variant in a mosaic state in his father. In fact, reanalysis of the parental data visualizing the BAM file detected this variant in two out of 50 reads. This finding was confirmed in the father using a custom next-generation sequencing clinical panel containing 1,253 genes involved in intellectual disability, autism spectrum disorders, and other common genetic disorders (Clinical panel V1.0). The variant was found again in 2 of 50 reads, and the mutant allele fraction was estimated to be around $4 \%$. In this case, the low somatic mosaicism detected in the father allowed the identification of the variant in the proband when filtering by de novo variants because the genotyping tool used to obtain the variants (GATK HaplotypeCaller) establishes a cut-off of $10 \%$ of reads to call a de novo allele. At the time of diagnosis the father was expecting a baby with a different partner. The recurrence risk of 1 to $2 \%$ in the case of a de novo mutation due to the possibility of parental gonadal mosaicism substantially increased to 10 to $20 \%$ in view of MAGEL2 being an imprinted gene and the presence of mosaicism in paternal tissues.

In conclusion, we report on another case of molecularly confirmed Schaaf-Yang syndrome, which - to the best of our knowledge-is the first report of a MAGEL2 mutation inherited from a mosaic father. We also highlight the difficulties encountered in analyzing single patients when the causal variant is located in an imprinted gene or a parental mosaicism is present. Generally, trio-based analysis is a good approach in cases of unexplained ID/CM when a de novo variant is suspected; however, as illustrated by this case, a specific analysis pipeline for imprinted genes that does not include inheritance filtering should also be considered. To this extent de novo, inherited variants, and variants present in a mosaic state in the parents will be detected. In cases where the causative mutation is located in a non-imprinted gene, low parental mosaicism may be missed when applying a de novo filtering strategy and might be subsequently suspected in the direct visualization of the trio BAM file and/or during Sanger sequencing confirmation. Meanwhile, a pathogenic dominant mutation in a proband may be overlooked if parental mosaicism is present in a higher percentage and a common de novo filtering strategy is applied. Therefore, we also recommend a specific bioinformatic algorithm for imprinted genes, and raise awareness of parental mosaicism as a possible pitfall in routine de novo analysis and its implications in genetic counseling.

\section{DISCLOSURE}

The authors declare no conflict of interest. 


\section{ACKNOWLEDGMENTS}

This study is supported by FIS grants from the Instituto de Salud Carlos III: PI13/02010 and PI14/1922.

María Palomares-Bralo, $P h D^{1,2}$, Elena Vallespín, $P h D^{1,2}$, Ángela del Pozo, $M S c^{1,2}$, Kristina Ibañez, $M S c, P h D^{1,2}$, Juan Carlos Silla, $M S c^{1,2}$, Enrique Galán, $M D, P h D^{2,3}$, Gema Gordo, BSc ${ }^{1,2}$, Victor Martínez-Glez, $\mathrm{MD}, \mathrm{PhD}^{1,2}$, Lázaro I. Alba-Valdivia, BSc ${ }^{1}$, Karen E Heath, $P h D^{1,2}$, Sixto García-Miñaúr, $M D^{1,2}$, Pablo Lapunzina, $M D, P h D^{1,2}$ and Fernando Santos-Simarro, $M D^{1,2}$

${ }^{1}$ Instituto de Genética Médica y Molecular (INGEMM), Hospital Universitario La Paz, IdiPaz, Madrid, Spain; ${ }^{2}$ CIBERER, Centro de Investigación Biomédica en Red de
Enfermedades Raras, ISCIII, Madrid, Spain; ${ }^{3}$ Departamento de Pediatria, Hospital Materno Infantil, Badajoz, Spain. Correspondence: Fernando Santos-Simarro

(fernando.santos@salud.madrid.org)

\section{REFERENCES}

1. Deciphering Developmental Disorders Study. Prevalence, phenotype and architecture of developmental disorders caused by de novo mutation. Nature 2017:542:433-438.

2. Aten $E$, Fountain $M D$, van Haeringen $A$, et al. Imprinting: the Achilles heel of trio-based exome sequencing. Genet Med 2016;18:1163-1164.

3. Schaaf CP, Gonzalez-Garay ML, Xia F, et al. Truncating mutations of MAGEL2 cause Prader-Willi phenotypes and autism. Nat Genet 2013;45: $1405-1408$.

Advance online publication 22 June 2017. doi:10.1038/gim.2017.42 